\title{
Impact of TRT and HRT on Parkinson onset in men and women.
}

\author{
Edoardo Cervoni ${ }^{1}$ \\ ${ }^{1}$ Locumdoctor $4 \mathrm{u}$
}

June 12, 2020

\begin{abstract}
INTRODUCTION: PD is more common with increasing age and shows male predominance, which is more obvious in Western studies. The reasons of this remain obscure. However, it has been suggested that exposure to endogenous and exogenous oestrogen contributes to these sex differences and that oestrogen prevents dopaminergic neuron depletion induced by neurotoxins in and therefore is neuroprotective. OBJECTIVES: we wanted to investigate if the exogenous administration of testosterone in males, and oestrogen with or without progesterone in females, had any protective effect towards to PD. METHODS: clinical records of 99929 residents in North West England were scrutinised in primary care setting for diagnosis of PD, HRT, and timing of onset in those cases were the adverse event had been identified.RESULTS: PD overall prevalence was of $0.55 \%$ in males and $0.36 \%$ in females $(\mathrm{M} / \mathrm{F}=3: 2)$. The NNT for HRT with testosterone was 1.016 (harm) significance level $\mathrm{P}<0.0001$, and NNT for HRT oestrogen+/-progesterone was 17.092 (harm) significance level $\mathrm{P}<0.0001$.CONCLUSIONS: HRT for males or females have not demonstrated any beneficial effects in terms of PD onset prevention or procrastination. On the contrary HRT, particularly with testosterone, is more likely to have a detrimental effect. Our results need to be interpreted cautiously as the treatment with HRT was not randomized and it cannot be excluded that stopping HRT may cause a sudden hormonal drop which in turn may have a negative effect.
\end{abstract}

\section{Introduction}

Parkinson disease is one of the major neurodegenerative diseases of our century and is characterized by progressive neuronal degeneration. This degeneration occurs in the substantia nigra, where the production of dopamine decreases consistently. Accumulations of $\alpha$-synuclein begin to appear spreading the disease throughout the brain. (K Taguchi)

Although pathogenic mechanisms are not yet known, it seems that there are several factors at play, among which the most relevant are genetic mutations, toxic environmental factors that inhibit the activity of the mitochondrial complex, microglial activation and inflammation, impaired autophagy, and the neural damage associated with ageing more in general.

Epidemiological studies have revealed a high male prevalence in Parkinson. A study in which 297 patients (181 males and 116 women) were evaluated using the Unified Parkinson Disease Rating Scale (UPDRS) showed that even the clinical progression of the disease can be significantly faster in men than in women $(\mathrm{H}$ Sawada).

Although the reason for this gender difference is not yet known, it would seem that oestrogens can play an important protective role against dopaminergic neuronal degeneration. 
A series of studies that have investigated the neuroprotective mechanisms of oestrogens, have revealed complex mechanisms that include the antioxidant properties of oestrogens and their ability to adjust adaptively the genes associated with apoptosis

(Petrovska S).

Clinical and the experimental evidence indicate a modulatory role of oestrogens on the activity of the nigrostriatal dopaminergic neurons. However, conflicting data make the precise effect of oestrogen on the nigrostriatal dopaminergic neurons unclear suggesting either a facilitator, or inhibitory role.

Use of HRT is widespread in general practice and, more recently, also testosterone replacement therapy is becoming more frequent among the male population.

We wanted to investigate, retrospectively, if the exogenous administration of testosterone in males, and oestrogen with or without progesterone in females, for reasons other than $\mathrm{PD}$, had protective, neutral, or negative effect, towards to developing PD later in life.

\section{Methods}

This is a large retrospective cohort study.

Clinical records of 99929 residents in North West England were scrutinised in primary care setting for diagnosis of PD, HRT, and timing of onset in those cases were the adverse event had been identified.

\section{Results}

Of the base population, 47164 were males and 54901 were females. Of them, respectively 47068 and 52861 never received male or female HRT. HRT treatment was therefore significantly more frequent in women.

Parkinson disease was diagnosed in 261 males and 196 females.

Of them, 1 man had received TRT and 13 women HRT.

To summarise, $\mathrm{PD}$ overall prevalence was of $0.55 \%$ in males and $0.36 \%$ in females $(\mathrm{M} / \mathrm{F}=3: 2)$. The NNT for HRT with testosterone was 1.016 (harm) significance level $\mathrm{P}<0.0001$, and NNT for HRT oestrogen+/progesterone was 17.092 (harm) significance level $\mathrm{P}<0.0001$. 


\begin{tabular}{|l|l|}
\hline Relative risk & 180.1542 \\
\hline $95 \% \mathrm{Cl}$ & 159.3182 to 203.7151 \\
\hline $\mathrm{z}$ statistic & 82.824 \\
\hline Significance level & $\mathrm{P}<0.0001$ \\
\hline NNT (Harm) & 1.016 \\
\hline $95 \% \mathrm{Cl}$ & 1.032 (Harm) to 1.001 (Harm) \\
\hline
\end{tabular}

Figure 1: Males Testosterone + Parkinson

\begin{tabular}{|l|l|}
\hline Relative risk & 16.8377 \\
\hline $95 \% \mathrm{Cl}$ & 9.7665 to 29.0286 \\
\hline z statistic & 10.161 \\
\hline Significance level & $\mathrm{P}<0.0001$ \\
\hline NNT (Harm) & 17.092 \\
\hline $95 \% \mathrm{Cl}$ & 19.988 (Harm) to 14.929 (Harm) \\
\hline
\end{tabular}

Figure 2: Female PD and HRT

\begin{tabular}{|l|l|}
\hline Difference & $54.64 \%$ \\
\hline $95 \% \mathrm{Cl}$ & 54.1874 to 55.0910 \\
\hline Chi-squared & 39757.399 \\
\hline DF & 1 \\
\hline Significance level & $\mathrm{P}<0.0001$ \\
\hline
\end{tabular}

Figure 3: Comparison of proportions 


\section{Discussion}

This is the largest study in its kind. However, due to its limitations, it cannot offer a definite answer about the clinical effect of sexual hormones on PD, even if it certainly appears to suggest that exogenous administration fails to promise a solution to the condition.

There are several design weaknesses.

Firstly, the latent period and the duration and specific type and dose of HRT, or TRT, could not have been established due to the study design.

Secondly, as far males are concerned, it cannot be excluded that those receiving TRT may have been harmed to start with by a clinical drop of the androgen levels, or by a baseline deprivation, absolute or relative, of androgens when compared to the rest of the male population.

Because the study was entirely anonymised, it did not look at the specific characteristics of the patients either. As it is well known that low testosterone levels are associated with other medical conditions, the greater incidence of PD in the TRT group could also have been linked to the latter issues.

As for the females, it could be argued that early ovarian failure leading to HRT supplementation, or a sudden drop leading to HRT request due to more severe symptoms, could be playing a factor in the development of PD. Furthermore, there is no information about the lifelong exposure to exogenous sexual hormones, for instance via oral contraceptives, in terms of presence, dose, and type.

The role of sex hormones in PD has been long debated.

Some Authors adamantly support a protective effect on the basis of studies highlighting that sex steroids have neuroprotective properties against various brain injuries. This paper reviews the protective effects of sex hormones, particularly oestradiol, progesterone and androgens, in the 1-methyl-4-phenyl-1,2,3,6tetrahydropyridine (MPTP) animal model of Parkinson's disease as compared to methamphetamine toxicity. (Bourque M)

The molecular mechanisms underlying beneficial actions of sex steroids on the brain have been investigated showing steroid, dose, timing and duration specificities and presently focus is on the dopamine signalling pathways the next frontier. Both genomic and non-genomic actions of oestrogen converge to promote survival factors and show sex differences. Neuroprotection by oestrogen involves activation of signaling molecules such as the phosphatidylinositol-3 kinase/Akt and the mitogen-activated protein kinase pathways. Interaction with growth factors, such as insuline-like growth factor 1, also contributes to protective actions of oestrogen,

Experimental data suggest a modulatory role of oestrogens on the activity of hypothalamic neurons.

Hypothalamic dopaminergic tonus influences the gonadotropin hypophiseal secretion, namely LH, by stimulating GnRH secretion from the median eminence and modulating the activity of the endogenous opioid system which in turn inhibits LH secretion.

There have been suggestions that the neurotransmitter mechanisms, which regulate LH secretion, are altered, and, in particular, the activity of the endogenous opioid system is deficient in women with PD. Thus, also the eventual "direction" of the level of female hormones remains uncertain.

Neurological diseases and neuropsychiatric disorders that vary depending on female life stages suggest that sex hormones may influence the function of neurotransmitter regulatory machinery such as the dopamine transporter.

Similar complexities are encountered when considering male hormones.

In rodents' nigrostriatal responsivity to dopamine may be modulated by testosterone acting via androgen receptors to alter gene expression of molecules involved in dopamine signalling during adolescence. (PurvesTyson TD) 
The mechanisms underlying this gender bias remain elusive, although one possibility is that androgens may increase dopamine neuronal vulnerability to oxidative stress. Motor impairment can be modelled in rats receiving a unilateral injection of 6-hydroxydopamine (6-OHDA), a neurotoxin producing nigrostriatal degeneration. To investigate the role of androgens in PD, we compared young (2 months) and aged (24 months) male rats receiving gonadectomy (GDX) and their corresponding intact controls. One month after GDX, rats were unilaterally injected with 6-OHDA, and their motor impairment and asymmetry were assessed 2 weeks later using the cylinder test and the amphetamine-induced rotation test. Plasma samples were also collected to assess the concentration of testosterone and advanced oxidation protein products, a product of oxidative stress. GDX decreased lesion-induced asymmetry along with oxidative stress and increased amphetamine-induced rotations. These results show that GDX improves motor behaviours by decreasing motor asymmetry in 6-OHDA-treated rats, an effect that may be ascribed to increased release of striatal dopamine and decreased oxidative stress.

Collectively, the data support the hypothesis that androgens may underlie the gender bias observed in PD.

Finally, it has been suggested that androgens induce dopaminergic neurotoxicity via caspase-3-dependent activation of protein kinase $\mathrm{C}$ delta.Â (Cunningham RL)

Albeit the number of males in the study receiving TRT was very small, there is no compelling evidence that TRT may have a protective role towards to protecting from PD later in life.

Also, HRT does not appear to have any protective effect in females.

\section{Conclusions}

TRT for and HRT for females have not demonstrated any beneficial effects in terms of PD onset prevention, or procrastination. On the contrary, particularly testosterone, is more likely to have a detrimental effect. Our results need to be interpreted cautiously as the treatment with HRT and TRT were not randomized and it cannot be excluded that stopping those treatments may cause a sudden hormonal drop which in turn may have a negative effect of some significance when considering the onset of PD.

\section{Bibliography}

1. Bourque M, Dluzen DE, Di Paolo T. "Neuroprotective actions of sex steroids in Parkisnon's disease." Front Neuroendocrinol (2009): 142-157.

2. Cunningham RL, Giuffrida A, Roberts JL. "Androgens induce dopaminergic neurotoxicity via caspase3-dependent activation of protein kinase Cdelta." Endocrinology (2009): 150(12):5539-5548.

3. H Sawada, S Shimohama. "Estrogens and Parkisnon Disease." Endocrine (2003): 77-79.

4. K Taguchi, Y Watanabe, A Tsujimura, M Tanaka. "Brain region-dependent differential expression of alpha-synuclein." J Comp Neurol (2016): 1236-1258.

5. Petrovska S, Dejanova B, Jurisic V. "Estrogens: mechanisms of neuroprotective effects." $J$ Physiol Biochem(2012): 455-460.

6. Purves-Tyson TD, Owens SJ, Double KL, Desai R, Handelsman DJ, Weickert CS. "Testosterone induces molecular changes in dopamine signaling pathway molecules in the adolescent male rat nigrostriatal pathway." PLoS One (2014): 9(3). 
